\title{
Características estomáticas de pares congenéricos de cerrado e mata de galeria crescendo numa região transicional no Brasil Central
}

\author{
Davi Rodrigo Rossatto ${ }^{1,3}$, William Arthur Hoffmann² e Augusto César Franco ${ }^{1}$
}

\author{
Recebido em 22/04/2008. Aceito em 6/10/2008
}

\begin{abstract}
RESUMO - (Características estomáticas de pares congenéricos de cerrado e mata de galeria crescendo numa região transicional no Brasil Central). Em áreas protegidas do fogo, espécies arbóreas predominantemente florestais conseguem se estabelecer no cerrado e crescer lado a lado com espécies do mesmo gênero que são características destas formações savânicas. Devido às condições ambientais diferenciadas encontradas nas formações de cerrado e de mata, estas espécies congenéricas podem se comportar como grupos funcionais distintos. Neste trabalho foi realizado um estudo comparativo da anatomia dos estômatos e da condutância estomática e taxas de transpiração foliar em 10 pares de espécies congenéricas do cerrado e de mata de galeria e das relações entre as características estomáticas selecionadas e a condutância estomática, já que a morfologia dos estômatos e sua densidade afetam a área para difusão dos gases e sua trajetória através dos poros estomáticos. Cada par foi de uma família diferente. Para a maioria das espécies, a área do poro estomático foi o fator de maior influencia no processo de trocas gasosas, pela sua correlação com a condutância estomática, enquanto a densidade estomática mostrou uma correlação negativa significativa tanto com o comprimento da célula-guarda quanto com a área do poro estomático. As espécies do cerrado apresentaram valores significativamente maiores do comprimento da célula-guarda e do poro estomático. No entanto, para a maioria dos outros parâmetros estomáticos examinados, a maior parte da variação pode ser atribuída a diferenças entre os gêneros, sendo que a variação entre as espécies dos dois ambientes parece ser um produto da historia evolutiva de cada grupo e não reflete uma especialização ao ambiente de cerrado ou de mata de galeria.
\end{abstract}

Palavras-chave: condutância estomática, estômato, poro estomático, transpiração

\begin{abstract}
Stomatal traits of cerrado and gallery forest congeneric pairs growing in a transitional region in Central Brazil). In fireprotected sites of Central Brazil, forest trees can establish in the neighbouring savanna and grow side by side with cerrado trees of the same genera. Because of the large differences in their native environment, the cerrado and forest species may represent two distinct functional types. In this study we performed a comparative study of stomata anatomical characteristics, stomatal conductance and transpiration of 10 congeneric species pairs of cerrado and gallery forest species. Each pair was from a different family. Because stomatal anatomy and density affect the area and the path for gas diffusion through the stomatal pores, we also assessed possible relationships among the selected stomata traits and stomatal conductance. The area of the stomatal pore was the anatomical trait with the largest influence on gas exchange. Increases in pore area were correlated with an increase in stomatal conductance for most species. On the other hand, stomatal density was negatively correlated with both the length of the guard-cell and the area of the stomatal pore. The length of the stomatal pore and of the guard cell was significantly greater for cerrado species. However, most of the observed variation could be attributed to differences among genera, indicating that these stomatal traits are the product of the particular evolutionary history of each genus, and not the result of specialization to the cerrado or forest environment.
\end{abstract}

Key words: stomata, stomatal conductance, stomatal pore, transpiration

\section{Introdução}

O Cerrado brasileiro abrange uma área aproximada de 2 milhões de $\mathrm{km}^{2}$, correspondendo a $22 \%$ do território nacional. Por ocorrer ao longo de uma ampla faixa latitudinal está submetido a diferenças de clima, relevo e de solos, além de apresentar uma grande variedade de fitofisionomias e uma alta diversidade vegetal (Oliveira Filho \& Ratter 2002). Os cerrados estão submetidos a uma extrema sazonalidade pluviométrica; os verões (outubro a abril) são quentes e chuvosos e os invernos (maio a setembro) são frios e secos. Os cerrados brasileiros também são caracterizados pela baixa disponibilidade nutricional no solo (Goodland \& Pollard 1973) e pela presença freqüente de fogo (Coutinho 1978), sendo estes estresses marcantes na determinação das fitofisionomias, da composição florística e das adaptações funcionais.

Em muitos locais do cerrado existem regiões ecotonais entre as formações savânicas e florestais. A

\footnotetext{
1 Universidade de Brasília, Instituto de Biociências Biológicas, Departamento de Botânica, Laboratório de Fisiologia Vegetal, C. Postal 04457, 70904-970 Brasília, DF, Brasil

2 North Carolina State University, Department of Plant Biology, Campus Box 7612, Raleigh, NC, 27695-7612, USA

3 Autor para correspondência: drrossatto@unb.br
} 
transição entre estas duas fisionomias geralmente é abrupta (Cole 1992), sendo caracterizada por diferenças na densidade arbórea e também na composição de espécies (Felfili \& Silva Júnior 1992). No entanto, apesar das diferenças na composição de espécies, é comum a ocorrência de pares congenéricos nas regiões de transição entre as duas fitofisionomias, ou seja, a ocorrência de duas espécies do mesmo gênero, em que uma é típica da formação savânica enquanto a outra é encontrada predominantemente na formação florestal. Estas espécies, muitas vezes, são encontradas lado a lado nestas regiões de transição, mostrando assim que algumas espécies florestais têm a capacidade de se estabelecer e colonizar as formações savânicas circundantes (Hoffmann et al. 2004).

A transição entre cerrado e floresta representa o limite natural da distribuição das florestas tropicais, desta forma o estudo das diferenças fisiológicas entre as espécies ali presentes é de vital importância para o correto entendimento dos fatores ambientais e atributos da vegetação que determinam a ocorrência destes ecótonos e para realizar prognósticos sobre a distribuição da vegetação em resposta às mudanças climáticas e regimes de distúrbio (Hoffmann 2000). A expansão da floresta em direção a regiões savânicas do Centro-Oeste seria a resposta esperada durante períodos mais úmidos, como ocorreu diversas vezes durante o Pleistoceno (Ledru 2002) e que continua ocorrendo atualmente (Silva et al. 2008).

Desta maneira, para compreensão desta zona de equilíbrio dinâmico que se estabelece entre o cerrado e a floresta é fundamental o estudo das características adaptativas das espécies florestais em regiões de cerrado (Hoffmann et al. 2004). Além disto, através da comparação entre pares congenéricos de cerrado e de floresta pode-se esclarecer diferenças adaptativas que surgiram independentemente entre taxas não relacionados, examinando-se as espécies através de uma perspectiva evolutiva, controlando o componente filogenético (Hoffmann \& Franco 2003).

Segundo Pillar \& Sosinski Jr. (2003) um grupo funcional é caracterizado por um conjunto de espécies vegetais, que independentemente de sua filogenia, são similares em algumas características, apresentando padrões similares de associação com certas variáveis. Devido às características diferenciadas entre as formações de cerrado e as formações florestais, podese postular que as espécies de cerrado e de mata se comportariam como grupos funcionais distintos, devido às claras diferenças ambientais encontradas entre estes ambientes (Hoffmann et al. 2005). Desta maneira a fisiologia das espécies de cerrado estaria adaptada a responder ao estresse hídrico sazonal, altas intensidades luminosas e deficiência nutricional (Franco 2002), enquanto espécies florestais apresentariam adaptações relacionadas à tolerância ao sombreamento, pois a luz é o principal fator limitante nestas formações (Carswell et al. 2000). Os estudos com os pares congenéricos têm mostrado algumas diferenças entre espécies do cerrado e de mata em características funcionais foliares relacionadas à produtividade, como área foliar específica e concentração de nutrientes foliares (Hoffmann et al. 2005).

As características morfo-anatômicas das plantas são grandemente influenciadas pelos fatores ambientais, estando associadas principalmente com o regime de luz, quantidade de chuva, tipo de solo e altitude (Givnish 1984). Estes fatores variam no espaço e no tempo e podem atuar como limitantes para o estabelecimento e crescimento das diferentes espécies vegetais. As folhas são os órgãos vegetais mais expostos ao meio ambiente circundante e suas modificações têm sido interpretadas como respostas adaptativas a mudanças nas condições ambientais (Fahn 1986). Estudos anatômicos têm mostrado uma grande variação na estrutura foliar de espécies lenhosas do cerrado, principalmente em resposta as variações da incidência luminosa (Marques et al. 2000), ao conteúdo de nutrientes no solo e aos efeitos da sazonalidade (Justo et al. 2005). Estas mudanças na estrutura foliar podem afetar a economia hídrica e a fixação de carbono.

Segundo Franco (2002), o sucesso de uma espécie vegetal encontrada num ambiente sazonal depende, principalmente, da capacidade desta em manter um balanço hídrico adequado durante o período da seca, minimizando assim os efeitos que os déficits hídricos possam causar no balanço de carbono. Os estômatos têm um papel preponderante neste processo, ao regular simultaneamente o fluxo transpiratório para a atmosfera e o fluxo de $\mathrm{CO}_{2}$ para o interior da folha. A densidade estomática, o grau de abertura do poro estomático e a sua regulação são de extrema importância para a adaptação de espécies em regiões semi-áridas ou em ambientes sazonais, onde há uma estação seca claramente definida (Pearce et al. 2006). Estudos sobre os padrões de densidade de estômatos têm encontrado uma maior densidade estomática quando as folhas são expostas à alta irradiação solar ou a baixa disponibilidade hídrica, que estaria relacionado a um melhor controle da condutância estomática, propiciando um aumento na capacidade de regular as perdas de água por transpiração (Lleras 1974). Os parâmetros morfológicos dos estômatos, incluindo tamanho, freqüência e distribuição variam enormemente entre as diferentes espécies arbóreas de um mesmo ambiente (Boeger \& Wisniewski 2003) e também entre ambientes (Pandey \& Nagar 2003; 
Pearce et al. 2006), mostrando assim que as plantas possuem a capacidade de ajustar estas características em resposta às pressões ambientais presentes no momento em que a folha está em formação (Abrams et al. 1994).

Deste modo, estudos da anatomia do estomato em conjunto com as respostas fisiológicas podem mostrar evidência de aclimatação adaptativa a determinados ambientes (Smith et al. 1998; Sparks \& Black 1999; Dunlapp \& Stettler 2001). Assim sendo, pode-se postular que espécies florestais devem apresentar características estomáticas diferentes das espécies do mesmo gênero típicas do cerrado, devido a diferentes pressões seletivas encontradas em seus ambientes naturais de ocorrência. Espécies de cerrado devem apresentar uma maior densidade estomática, porém estômatos menores; características adaptativas relacionadas a ambientes onde a sazonalidade pluviométrica é determinante (Pearce et al. 2006). Espera-se também encontrar relações entre as características morfológicas dos estômatos e a condutância estomática, já que a morfologia dos estômatos e sua densidade afetam a área para difusão dos gases e sua trajetória através dos poros estomáticos (Nobel 1999).

\section{Materiais e métodos}

Área de estudo - Este estudo foi realizado na Reserva Ecológica do IBGE (Recor), distante aproximadamente $35 \mathrm{~km}$ do centro de Brasília, DF, estando sua sede localizada na intersecção das coordenadas geográficas $15^{\circ} 56^{\prime} \mathrm{S}$ e $47^{\circ} 52^{\prime} \mathrm{W}$. A cobertura vegetal da Reserva é composta das diversas fisionomias encontradas tipicamente no bioma cerrado: campo limpo, campo sujo, cerrado sensu stricto, cerradão e mata de galeria (Oliveira \& Ratter 2002), apresentando algumas áreas de transição entre as formações savânicas e as formações florestais. As áreas utilizadas neste estudo compreendem transições entre cerrado sensu stricto e mata de galeria, onde é possível encontrar espécies da mata crescendo lado a lado com espécies do cerrado do mesmo gênero. Estes ambientes transicionais apresentam uma densidade de espécies arbóreas maior que a comumente encontrada em regiões de cerrado típico, tendo um estrato herbáceo menos representativo em termos de densidade (Silva et al. 2008). Neste estudo também foram utilizadas regiões típicas de cerrado sensu stricto próximas a região de transição, pois até mesmo nestes locais algumas espécies de mata de galeria também estão presentes.

Espécies selecionadas - Foram utilizados dez pares congenéricos, onde um é tipicamente classificado como de mata de galeria e o outro de cerrado (Tab. 1). Cinco indivíduos de cada espécie foram marcados nas áreas de estudo, totalizando 100 indivíduos.

Análise anatômica e fisiológica - Para a análise anatômica foram coletadas de cada indivíduo cinco folhas que estavam expostas a luminosidade na maior parte do dia, totalizando 25 folhas por espécie. Por motivos de padronização só foram coletadas folhas totalmente expandidas, estando aproximadamente no quarto nó. Todas as folhas foram coletadas no final do mês de novembro e início de dezembro/2006, durante a estação chuvosa; época em que todos os indivíduos já haviam cessado o processo de produção de novas folhas e de expansão e maturação foliar. Todas as folhas utilizadas neste estudo foram marcadas previamente quando estavam expandindo durante o mês de agosto/2006, durante a estação seca.

Após a coleta da folha, uma amostra do limbo foliar de aproximadamente $4 \mathrm{~cm}^{2}$ foi retirado entre a margem e a nervura principal na porção mediana da folha ou folíolo, sendo colocado em solução aquosa contendo álcool etílico a 70\%, até ser processado no laboratório. A dissociação das epidermes foliares foi realizada colocando a amostra coletada num frasco contendo uma

Tabela 1. Espécies estudadas na Reserva Ecológica do IBGE, DF, Brasil.

\begin{tabular}{lll}
\hline Espécies & Espécies & Famílias \\
\hline Cerrado & Mata de Galeria & \\
Byrsonima crassa Nied. & Byrsonima laxiflora Griseb. & Malpighiaceae \\
Guapira noxia Netto (Lundell) & Guapira areolata (Heimerl) Lundell & Nyctaginaceae \\
Hymenaea stigonocarpa Mart. ex Hayne & Hymenaea martiana Hayne & Fabaceae \\
Miconia pohliana Cogn. & Miconia cuspidata Naudin & Melastomataceae \\
Myrcia tomentosa (Aubl.) DC. & Myrcia rostrata DC. & Myrtaceae \\
Myrsine guianensis (Aubl.) Kuntze & Myrsine ferruginea (Ruiz \& Pav.) Spreng. & Myrsinaceae \\
Styrax ferrugineus Nees \& Mart. & Styrax pohlii A.DC. & Styracaceae \\
Symplocos rhamnifolia A.D.C. & Symplocos mosenii Brand & Symplocaceae \\
Tabebuia ochracea (Cham.) Standl. & Tabebuia impetiginosa Standl. & Bignoniaceae \\
Vochysia thyrsoidea Pohl. & Vochysia tucanorum Mart. & Vochysiaceae
\end{tabular}


solução $1: 1$ de ácido acético glacial e peróxido de hidrogênio (modificado de Franklin 1945). O frasco foi mantido em estufa a temperatura de $60^{\circ} \mathrm{C}$ por 12 horas, ou pelo tempo necessário para a total dissociação das epidermes. Após este processo as epidermes foram lavadas com água destilada, colocadas em lâmina e coradas com safranina. A montagem das lâminas foi realizada utilizando glicerina como meio de montagem. Seis parâmetros foram analisados: densidade estomática, densidade de tricomas (quando presentes), o comprimento das células-guarda estomáticas, o diâmetro equatorial dos estômatos, aqui denominado de largura dos estômatos, comprimento e largura do poro estomático. A área do poro estomático foi calculada para verificar a sua influência na densidade e condutância estomática. A área do poro foi considerada como a de uma elipse, sendo calculada como o produto da metade da largura do poro pela metade do comprimento do poro, multiplicado por $\pi$. Além destes parâmetros foi calculado o IAE - Índice de Área Estomática, multiplicando-se a média do comprimento estomático (em $\mathrm{mm}$ ) pela densidade estomática (Ashton \& Berlyn 1994). A contagem destes parâmetros foi realizada em microscópio óptico (Modelo Olympus CX31) acoplado a uma câmera digital (Modelo Olympus C-7070). O número de medidas do tamanho dos estômatos foi tomado de 500 estômatos para cada indivíduo amostrado, utilizando vinte campos por folha amostrada na lente objetiva de 10x. Para análise da densidade estomática e de tricomas de cada folha coletada, foram amostrados 20 campos definidos aleatoriamente, com a lente objetiva de 10x.

Durante a última semana do mês de novembro/2006 foram realizadas também medições de condutância estomática máxima (gs) e transpiração (E) nos mesmos indivíduos onde foram coletadas as folhas para a análise anatômica. As folhas utilizadas nesta análise expandiram na mesma época das folhas coletadas para análise anatômica, tendo a mesma idade foliar. Os dados de gs e E foram obtidos em campo com o auxílio de um sistema aberto e portátil de trocas gasosas por infravermelho (LCA-4, Analytical Development Co., Hoddesdon, U.K.), sendo medidos 2 pares congenéricos por dia. As medidas foram tomadas sob uma densidade saturante de fluxo de fótons (entre 1200-1300 $\mu \mathrm{mol} \mathrm{m} \mathrm{m}^{-2} \mathrm{~s}^{-1}$ ) obtido com o auxílio de uma fonte de luz halógena acoplada à câmara do LCA-4. As medidas foram obtidas sempre durante o período das 8:00 e 11:30 da manhã, com o intuito de evitar os baixos valores de déficit de pressão de vapor de água no início da manhã e seus altos valores após o meio dia, que poderiam afetar a condutância estomática (Prado et al. 2004). Foram amostradas três folhas por indivíduo, totalizando 150 folhas para as espécies de cerrado e 150 folhas para as espécies de mata de galeria.
As medidas foram tomadas após um tempo de espera de 3 minutos para a estabilização do sistema.

Análise estatística - Na análise dos parâmetros anatômicos e fisiológicos foi utilizada a análise estatística ANOVA fatorial para testar os efeitos dos gêneros e do grupo funcional (espécie de cerrado $\times$ espécie de mata), sendo o gênero tratado como fator aleatório e o grupo funcional como fator fixo. Este tipo de análise foi utilizado com sucesso em outros trabalhos de pares congenéricos (Hoffmann \& Franco 2003; Hoffmann et al. 2005), pois analisa os dados através de uma perspectiva filogenética, separando os efeitos da variação entre grupo funcional da variação entre o gênero. Um efeito apenas do grupo funcional é indicativo de que os fatores atuantes nas diferenças são majoritariamente ambientais, enquanto um efeito apenas de gênero indicaria uma maior relação filogenética. A interação entre grupo funcional e gênero indica um efeito dos dois fatores e esta comparação é realizada par a par como se fosse um teste t pareado (Hoffmann et al. 2004). As correlações entre densidade estomática e comprimento da célula guarda foram realizadas através do coeficiente de correlação de Spearman (Zar 1999), utilizando-se as médias dos valores dos indivíduos. As relações entre área do poro estomático e densidade estomática e área do poro e condutância estomática foram realizadas no programa SigmaPlot 10 através de uma regressão linear simples (Zar 1999), utilizando a média dos valores para cada espécie. Nesta análise as espécies Vochysia tucanorum e Guapira noxia foram omitidas da análise. Para todas as análises foi adotado nível de significância a 5\%.

\section{Resultados}

Densidade estomática - Em todos os gêneros analisados os estômatos encontravam-se apenas na face abaxial, com exceção da espécie do cerrado H. stigonocarpa, que apresentou estômatos também na face adaxial. Neste caso os estômatos foram mais numerosos na face abaxial, sendo a espécie anfi-hipoestomática, com uma razão média de 7,47 $\pm 2,28$ (média \pm desvio padrão) estômatos na face abaxial, em relação aos estômatos na face adaxial.

A densidade estomática foi variável nos dois grupos funcionais (Fig. 1), entretanto não houve efeito do grupo funcional para esta variável $(P=0,623)$, mostrando que as densidades estomáticas são similares entre os grupos. As espécies do cerrado apresentaram valores entre 107 e 493 estômatos por $\mathrm{mm}^{2}$ enquanto as de mata entre 109 e 633 estômatos por $\mathrm{mm}^{2}$. Há um claro efeito filogenético nesta variável $(P=0,027)$. O gênero com maior densidade estomática foi Myrcia, enquanto Guapira foi o gênero com menor densidade estomática 


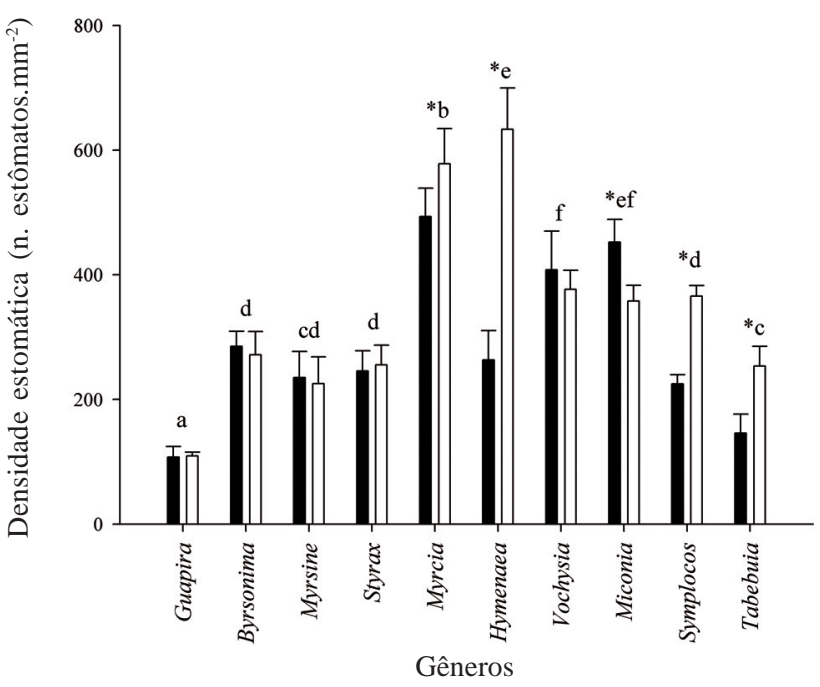

Figura 1. Densidade estomática para os pares congenéricos estudados. O símbolo * indica diferença entre espécies de um mesmo gênero $(P<0,05)$. Letras indicam diferenças entre os gêneros $(P<0,05)$. As barras verticais indicam o erro padrão. $(\boldsymbol{\square}=$ Cerrado; $\square=$ Mata $)$.

Os gêneros Byrsonima, Myrsine, Styrax e Symplocos apresentaram densidades estomáticas estatisticamente similares. Por outro lado, a interação grupo funcional $\times$ gênero foi significativa $(P=0,000)$, sendo que quatro espécies do grupo de mata (Myrcia, Hymenaea, Symplocos e Tabebuia) apresentaram maior densidade estomática do que as espécies dos mesmos gêneros no cerrado, enquanto Miconia apresentou o padrão inverso. Na Fig. 2 são mostrados exemplos de pares com densidade estomática semelhante (Myrsine, Guapira e Byrsonima) e também pares com diferenças significativas nas densidades estomáticas (Miconia e Symplocos).

Tamanho dos estômatos - Para o comprimento das células-guarda estomáticas houve efeito do grupo funcional $(P=0,013)$, tendo as espécies de cerrado maior comprimento do que as de mata (Fig. 3A). Houve efeito do gênero $(P=0,024)$, e da interação grupo funcional $\times$ gênero $(P=0,012)$, assim as espécies de cerrado dos gêneros Guapira, Myrcia, Hymenaea, Miconia, Symplocos e Tabebuia apresentaram célulasguardas maiores do que as espécies do mesmo gênero de mata. Para a largura dos estômatos (Fig. 3B), não foi encontrado efeito do grupo funcional $(P=0,654)$, mostrando semelhança nesta variável para os dois grupos. Foi encontrado apenas um efeito filogenético $(P=0,015)$, sendo Vochysia, Guapira e Tabebuia os gêneros com maior largura estomática.

Poro estomático - O comprimento do poro estomático variou entre 10 e $40 \mu \mathrm{m}$ (Fig. 4A). Para esta variável houve um efeito do grupo $(P=0,014)$ e da interação grupo funcional $\times$ gênero $(P=0,025)$, sendo que sete espécies do cerrado tiveram maior comprimento do poro estomático do que seus respectivos congenéricos de mata (Guapira, Hymenaea, Miconia, Myrcia, Symplocos, Tabebuia e Vochysia). Para a largura do poro estomático (Fig. 4B) houve apenas um efeito de gênero $(P=0,032)$ e da interação grupo x gênero $(P=0,011)$. Desta maneira,
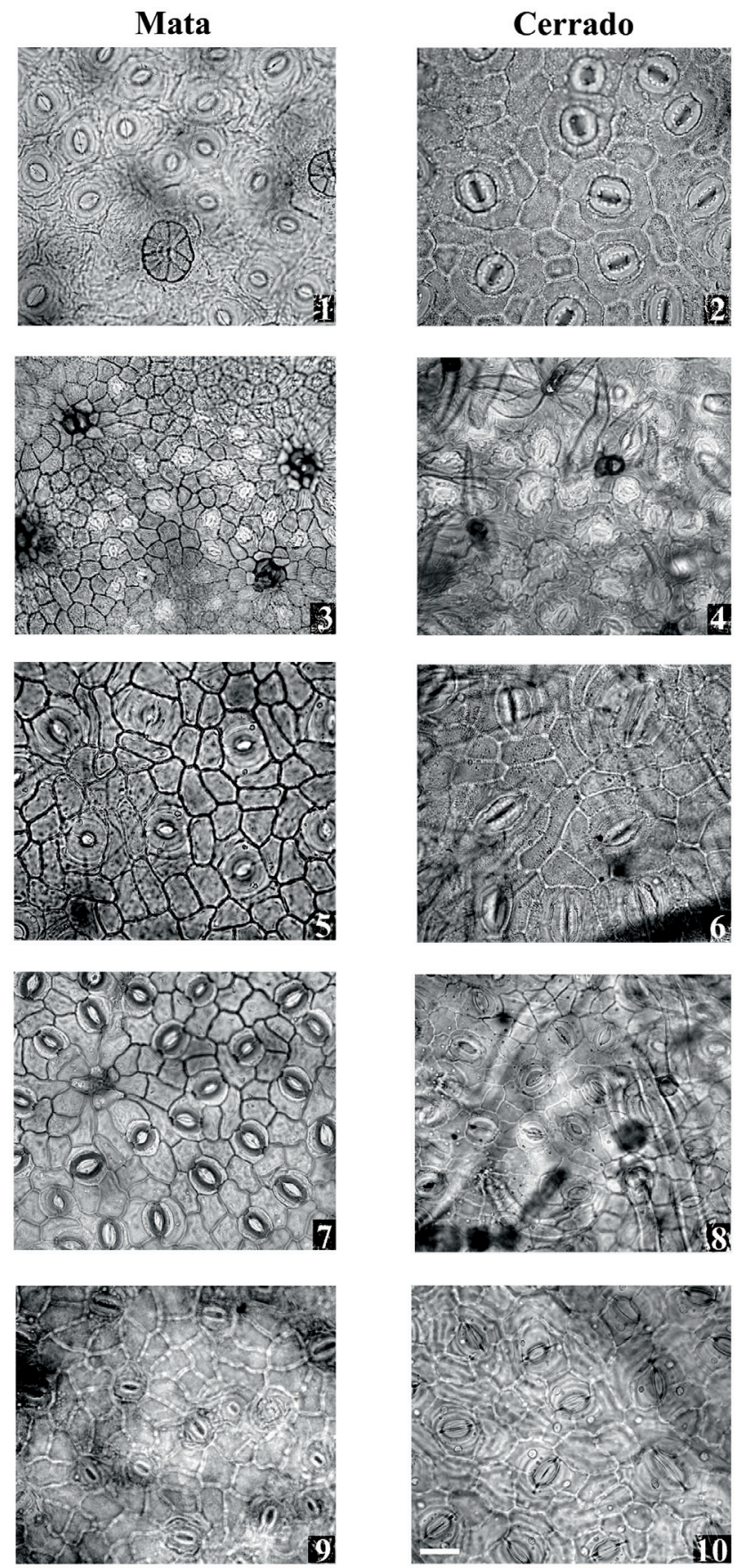

Figura 2. Padrões estomáticos para algumas espécies congenéricas de cerrado e mata de galeria. 1. Myrsine ferruginea. 2. Myrsine guianensis. 3. Miconia acuminata. 4. Miconia pohliana. 5. Guapira areolata. 6. Guapira noxia. 7. Byrsonima laxiflora. 8. Byrsonima crassa. 9. Symplocos mosenii. 10. Symplocos rhamnifolia. As figuras 1-2, 5-6 e 7-8 ilustram pares sem diferença na densidade estomática $(P>0,05)$. As figuras 3-4 e 9-10 ilustram pares com diferenças significativas na densidade estomática $(P<0,05)$. Barra: $20 \mu \mathrm{m}$. 


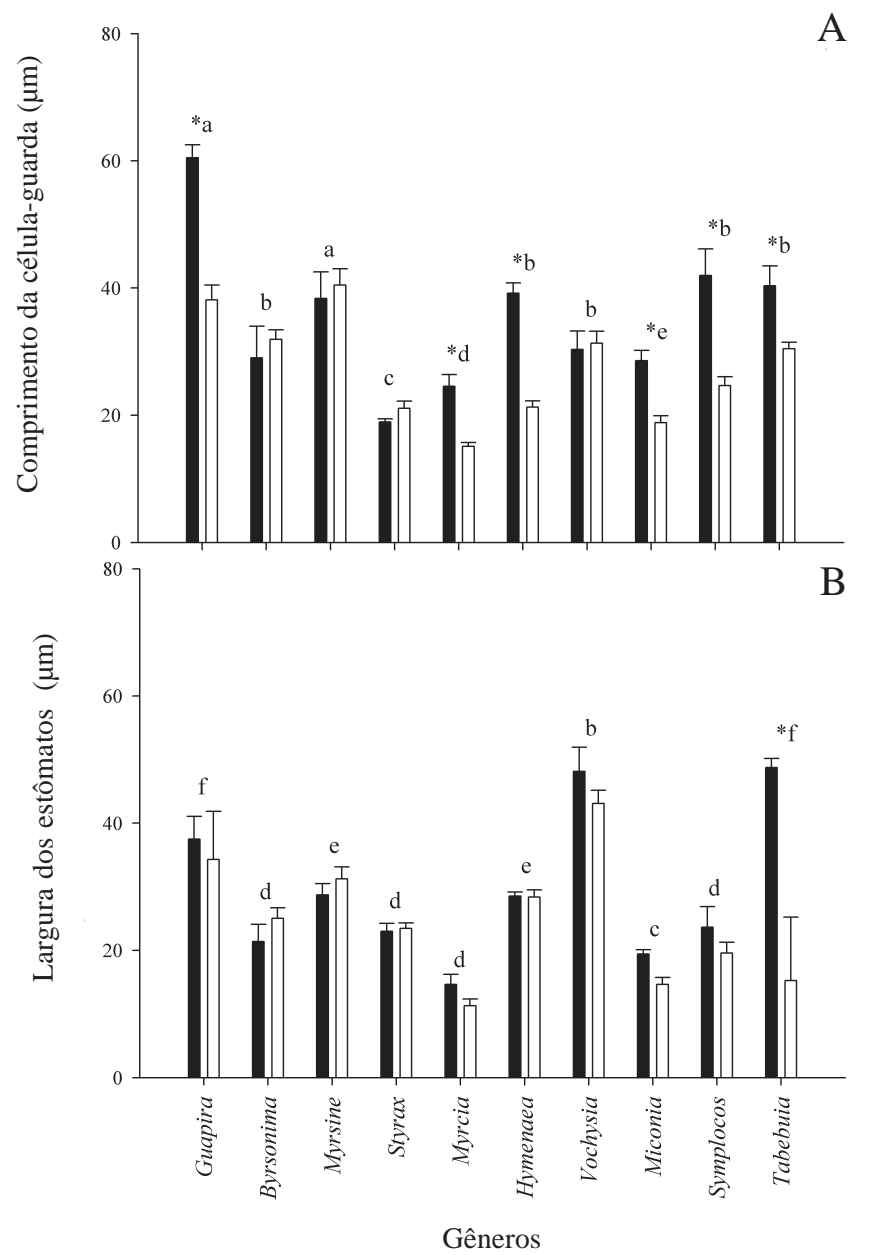

Figura 3. Comprimento da célula-guarda (A) e largura (B) dos estômatos dos pares congenéricos estudados. $\mathrm{O}$ símbolo * indica diferença entre espécies de um mesmo gênero $(P<0,05)$. Letras indicam diferenças entre os gêneros $(P<0,05)$. As barras verticais indicam o erro padrão. ( $\square=$ Cerrado; $\square=$ Mata $)$.

as espécies de cerrado de Guapira, Myrcia e Vochysia apresentaram maior largura do poro estomático em comparação à espécie da mata do mesmo gênero, enquanto a espécie de mata de Byrsonima apresentou maior largura do poro do que a do cerrado.

Índice estomático - Não há um efeito de grupo $(P=0,465)$ para esta variável, apenas um efeito de gênero $(P=0,037)$ e da interação grupo $\times$ gênero $(P=0,001)$. Assim só houve diferenças significativas entre as espécies dos gêneros Myrcia, Miconia e Hymenaea (Fig. 5), sendo os valores maiores para as espécies de cerrado dos dois primeiros gêneros, e para a espécie de mata do último.

Condutância estomática e transpiração - Os valores de condutância estomática e transpiração são mostrados na Tab. 2. A faixa de valores para a condutância esteve entre 60 e $200 \mathrm{mmol} . \mathrm{m}^{2} . \mathrm{s}^{-1}$ tanto para cerrado quanto para a mata, não havendo diferenças significativas entre os grupos funcionais $(P=0,904)$ e nem entre os gêneros

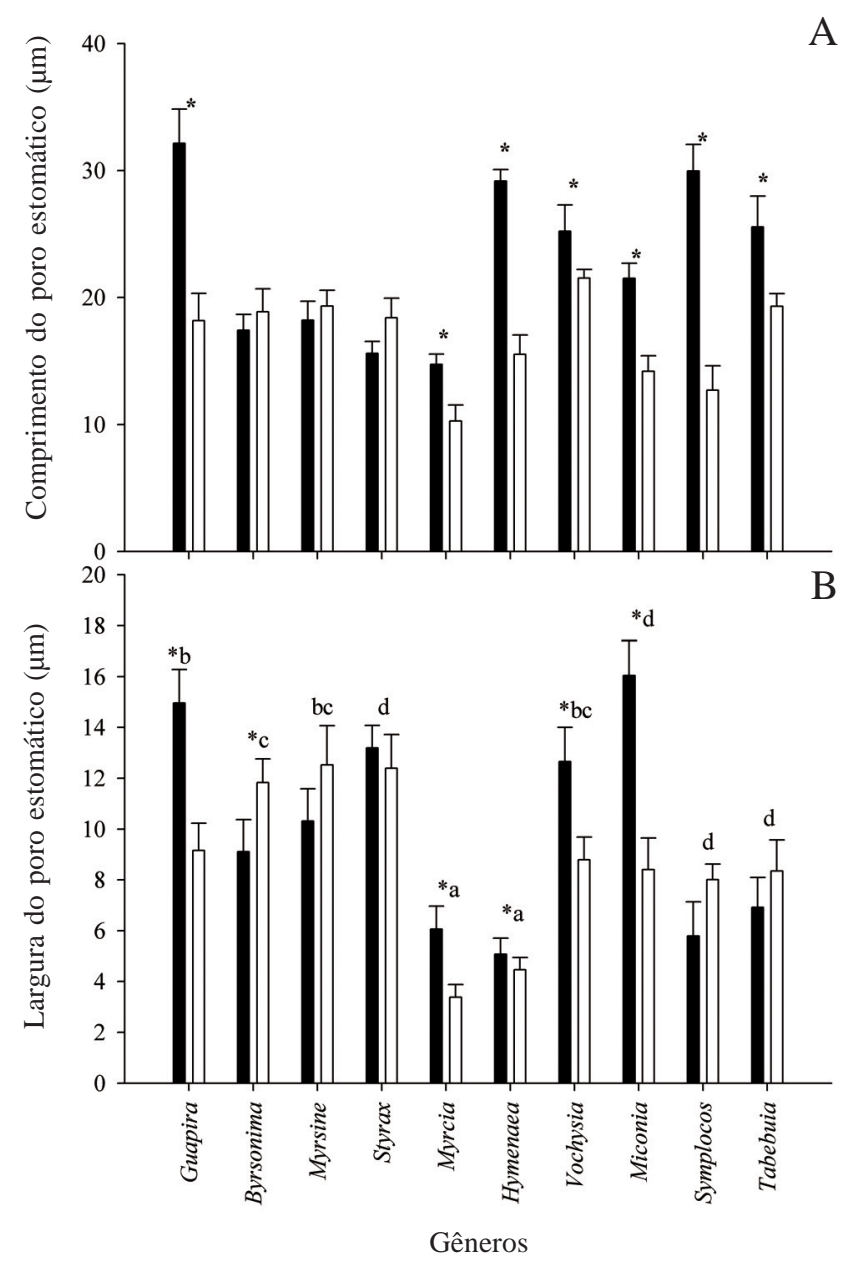

Figura 4. Comprimento (A) e largura (B) dos poros estomáticos dos pares congenéricos estudados. $\mathrm{O}$ símbolo $*$ indica diferença entre espécies de um mesmo gênero $(P<0,05)$. Letras indicam diferenças entre os gêneros $(P<0,05)$. As barras verticais indicam o erro padrão. ( $\square$ = Cerrado; $\square$ = Mata).

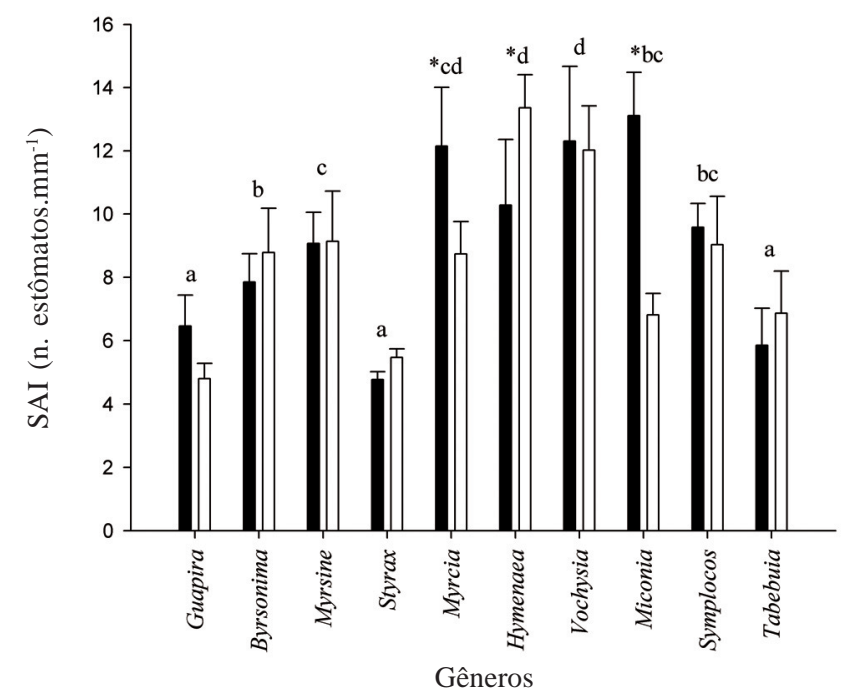

Figura 5. Índice de área estomática (IAE) para as espécies congenéricas estudadas. $\mathrm{O}$ símbolo * indica diferença entre espécies de um mesmo gênero $(P<0,05)$. Letras indicam diferenças entre os gêneros $(P<0,05)$. As barras verticais indicam o erro padrão. $(\square=$ Cerrado; $\square=$ Mata $)$. 
Tabela 2. Valores de condutância estomática máxima (gs) e taxas de transpiração (E) obtidas para os pares congenéricos estudados no mês de novembro/2006. Dados expressos em termos de média \pm erro padrão. Letras semelhantes indicam similaridade de acordo com o teste de Tukey $(\mathrm{p}>0,05)$

\begin{tabular}{llclc}
\hline & \multicolumn{2}{c}{ gs $\left(\mathrm{mmol} \cdot \mathrm{m}^{-2} \cdot \mathrm{s}^{-1}\right)$} & & $\mathrm{E}\left(\mathrm{mol} \cdot \mathrm{m}^{-2} \cdot \mathrm{s}^{-1}\right)$ \\
\cline { 2 - 3 } Gêneros/ GF & \multicolumn{1}{c}{ Cerrado } & Mata & Cerrado \\
\hline Guapira & $68,00(5,83) \mathrm{a}$ & $164,33(27,34) \mathrm{b}$ & $1,99(0,30) \mathrm{a}$ & $2,52(0,70) \mathrm{a}$ \\
Byrsonima & $97,67(8,78) \mathrm{a}$ & $206,67(10,21) \mathrm{b}$ & $1,71(0,32) \mathrm{a}$ & $2,82(0,19) \mathrm{a}$ \\
Myrsine & $216,00(19,58) \mathrm{a}$ & $127,60(13,90) \mathrm{b}$ & $5,58(0,43) \mathrm{a}$ & $2,83(0,65) \mathrm{b}$ \\
Styrax & $172,67(20,44) \mathrm{a}$ & $113,00(22,54) \mathrm{a}$ & $4,69(0,83) \mathrm{a}$ & $2,88(0,44) \mathrm{b}$ \\
Myrcia & $184,00(17,77) \mathrm{a}$ & $90,67(13,75) \mathrm{b}$ & $2,52(0,65) \mathrm{a}$ & $4,06(0,87) \mathrm{a}$ \\
Hymenaea & $165,33(32,57) \mathrm{a}$ & $98,00(16,14) \mathrm{a}$ & $2,34(0,47) \mathrm{a}$ & $3,11(0,83) \mathrm{a}$ \\
Vochysia & $107,00(6,63) \mathrm{a}$ & $203,00(13,19) \mathrm{b}$ & $2,19(0,25) \mathrm{a}$ & $4,22(0,49) \mathrm{b}$ \\
Miconia & $110,00(7,74) \mathrm{a}$ & $132,00(13,56) \mathrm{a}$ & $3,10(0,49) \mathrm{a}$ & $3,71(0,32) \mathrm{a}$ \\
Symplocos & $138,00(9,58) \mathrm{a}$ & $126,00(21,58) \mathrm{a}$ & $2,65(0,54) \mathrm{a}$ & $2,82(0,61) \mathrm{a}$ \\
Tabebuia & $140,02(13,50) \mathrm{a}$ & $106,33(23,29) \mathrm{a}$ & $2,68(0,45) \mathrm{a}$ & $2,74(0,61) \mathrm{a}$ \\
Média & $139,87(14,29) \mathrm{A}$ & $136,76(13,07) \mathrm{A}$ & $2,95(0,39) \mathrm{a}$ & $3,17(0,18) \mathrm{a}$ \\
\hline
\end{tabular}

$(P=0,984)$. Entretanto houve um efeito da interação grupo $\times$ gênero $(P=0,001)$, mostrando diferenças entre as espécies congenéricas em Guapira, Vochysia e Byrsonima , com as espécies de mata com maior valor de condutância estomática, e em Myrcia e Myrsine, com as espécies do cerrado com maiores valores (Tab. 2). Os valores de transpiração foram semelhantes para o grupo funcional $(P=0,144)$, porém houve um efeito da interação grupo funcional $\times$ gênero $(P=0,000)$, tendo as espécies do cerrado dos gêneros de Styrax e Myrsine maior transpiração, enquanto a espécie da mata do gênero Vochysia apresentou maior transpiração (Tab. 2).

Densidade de tricomas - Nenhuma das espécies estudadas apresentou tricomas na face adaxial, mas algumas apresentaram na face abaxial. Na Fig. 6 é possível observar os valores médios de densidade de tricomas para os gêneros analisados. No gênero Byrsonima, somente a espécie de cerrado apresentou tricomas (Fig. 2-4), enquanto nos gêneros Hymenaea e Tabebuia, somente as espécies de mata apresentaram tricomas. Tanto a espécie de mata como a de cerrado dos gêneros Styrax, Myrcia e Miconia apresentaram tricomas. A análise estatística para esses três gêneros não mostrou efeito do grupo $(P=0,864)$, nem efeito do gênero $(P=0,675)$, mas sim para a interação grupo funcional $\times$ gênero $(P=0,004)$, tendo a espécie da mata do gênero Myrcia uma maior densidade do que a de cerrado e a espécie de cerrado do gênero Miconia com maior densidade de tricomas do que a espécies de mata. As espécies de Styrax não diferiram na densidade de tricomas.

Relações entre as diferentes características estomáticas - Foi encontrada uma correlação negativa significativa $(\mathrm{r}=-0,66, P=0,004)$ entre densidade estomática e comprimento dos estômatos para o conjunto de espécies estudadas (Fig. 7), entretanto a densidade estomática não foi relacionada nem com a condutância $(P>0,20)$ nem com a transpiração $(\mathrm{P}>0,10)$. Houve uma clara relação entre a área do poro e a densidade de estômatos (Fig. $8 \mathrm{~A})$ para o conjunto de espécies $\left(\mathrm{r}^{2}=0,52, P=0,000\right)$, desde que Guapira noxia e Vochysia thyrsoidea sejam excluídas da análise. A faixa de variação da densidade estomática foi muito semelhante, porém houve diferenças na variação da área do poro estomático, estando entre 20 e $200 \mu \mathrm{m}^{2}$ para o grupo de mata e entre 60 e $420 \mu \mathrm{m}^{2}$ para o grupo de cerrado (Fig. 8A). A relação entre condutância estomática e área do poro estomático (Figura 8B) também foram significativas para as espécies estudadas $\left(\mathrm{r}^{2}=0,25, P=0,0379\right)$, desde que novamente

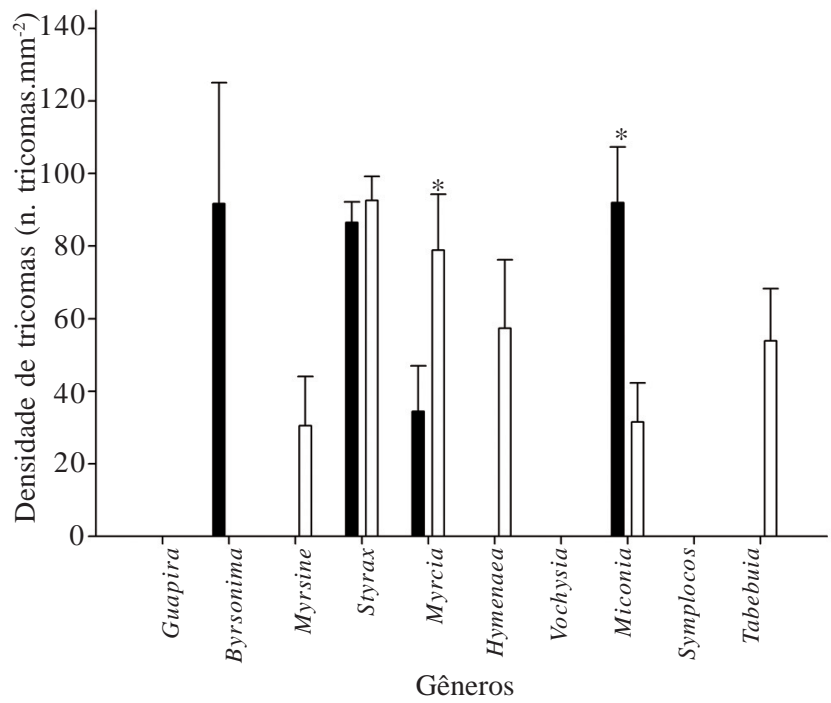

Figura 6. Densidade de tricomas para algumas espécies estudadas. O símbolo * indica diferença entre espécies de um mesmo gênero $(P<0,05)$. As barras verticais indicam o erro padrão. ( $\square=$ Cerrado; $\square=$ Mata). 


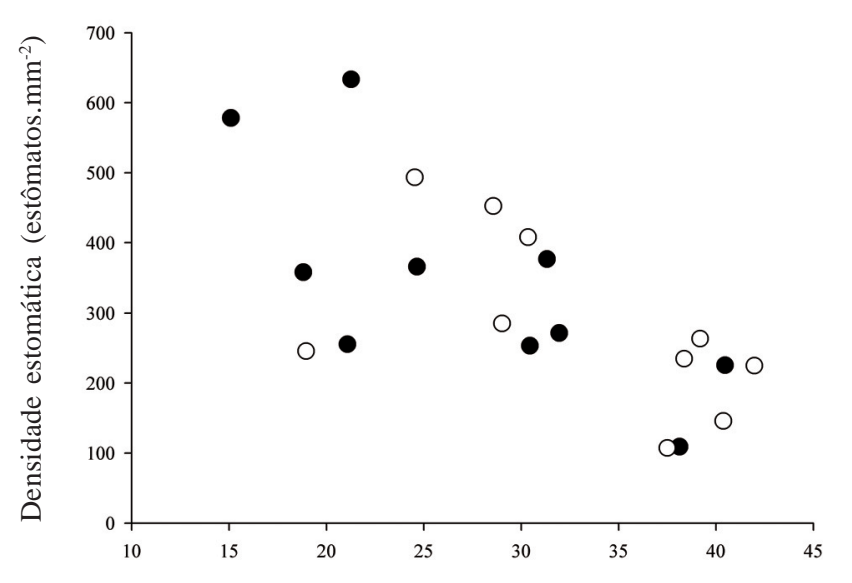

Comprimento dos estômatos $(\mu \mathrm{m})$

Figura 7. Relação ( $r=-0,66, P=0,004)$ entre comprimento dos estômatos e densidade estomática para o conjunto de espécies do cerrado e de mata $(\bullet$ Mata; $\bigcirc=$ Cerrado $)$.

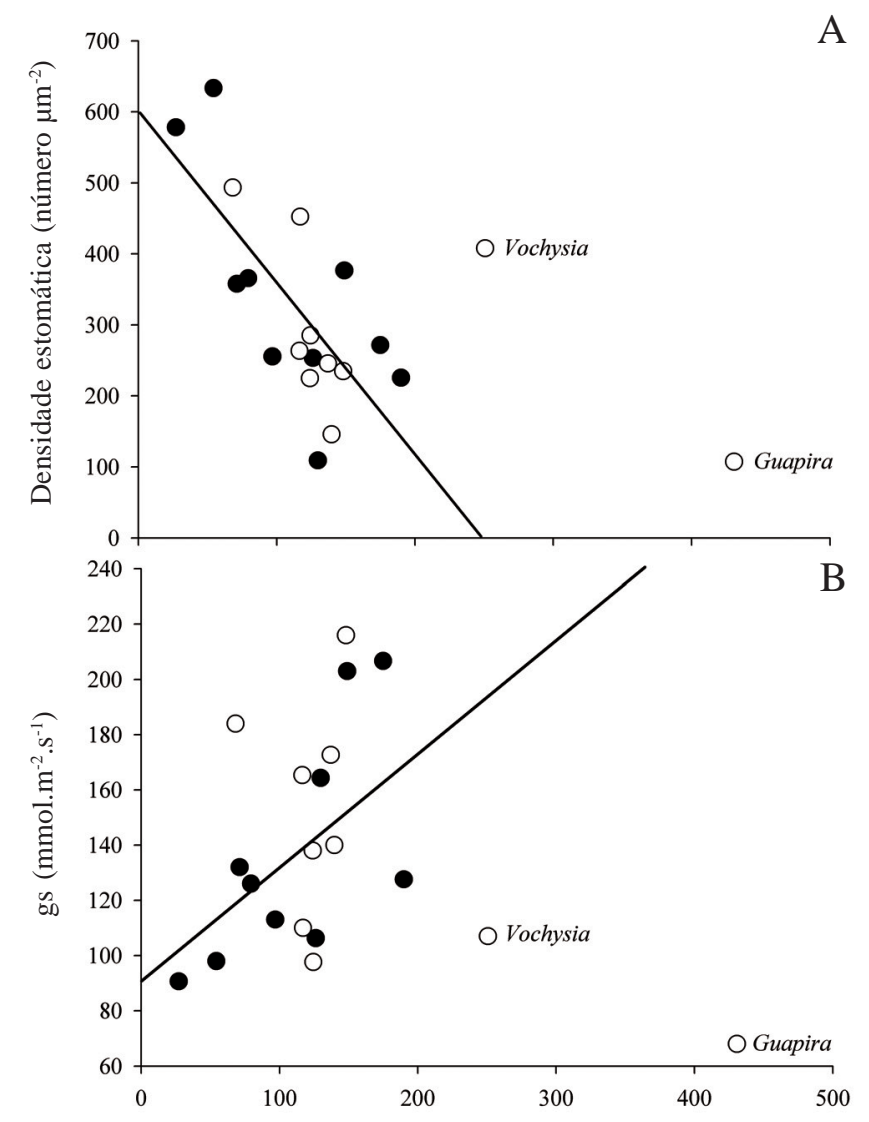

Área do poro estomático $(\mu \mathrm{m})$

Figura 8. Relação entre área do poro estomático e densidade estomática (A) e entre a área do poro estomático e a condutância estomática (B) para o conjunto de espécies do cerrado e de mata. Linha sólida é a reta ajustada aos dados por regressão linear $\left(\mathrm{A}-\mathrm{Y}=89,95+0,11 \mathrm{x}, \mathrm{r}^{2}=0,24, P=0,0379 ; \mathrm{B}-\mathrm{Y}=602,04-0,61 \mathrm{x}\right.$, $\left.\mathrm{r}^{2}=0,52, P=0,006\right)$. Vochysia thyrsoidea e Guapira noxia não foram incluídas na análise $(\bullet=$ Mata; $\bigcirc=$ Cerrado).
G. noxia e V. thyrsoidea fossem excluídas da análise. Essa relação não foi significativa para a transpiração $(P>0,05)$.

\section{Discussão}

Os valores encontrados para densidade estomática foram semelhantes entre os dois grupos funcionais, estando entre 107 e 633 estômatos $/ \mathrm{mm}^{2}$. Altas densidades estomáticas geralmente aparecem em ambientes mais sazonais, estando relacionadas à redução na disponibilidade hídrica (Pearce et al. 2006). A relação negativa entre comprimento da célula guarda e densidade estomática foi significativa e semelhante entre os dois grupos, sendo comumente relatada para diversas espécies em diferentes ambientes (Mishra 1997; Larcher 2000; Hetherington \& Woodward 2003).

Diferentemente do esperado, as espécies do cerrado apresentaram estômatos com células guarda maiores, e consequentemente maiores dimensões do poro estomático, em relação a espécies de mata de galeria. Estômatos maiores geralmente são característicos de ambientes mesofíticos, propiciando um consumo luxurioso de água (Galmes et al. 2007), enquanto estômatos menores seriam encontrados em ambientes xéricos, propiciando um controle hídrico mais adequado (Aasamaa et al. 2001). A presença de estômatos menores é comum em condições de alta luminosidade e baixa umidade do ar (Shields 1950), fatores atuantes no cerrado. A presença de estômatos maiores nas espécies deste grupo, porém, pode estar relacionada com o fato de que muitas espécies arbóreas no cerrado apresentam sistemas radiculares profundos (Rawitscher 1948; Jackson et al. 1999), tendo potencial para a captação de água das regiões mais profundas que permanecem úmidas mesmo durante a estação seca (Scholz et al. 2002). Algumas espécies do cerrado também apresentam transpiração noturna (Goldstein et al. 2008), que poderia ser advinda do fato de apresentarem poros estomáticos de grandes dimensões.

O aumento nos valores de condutância estomática geralmente está relacionado com aumentos no tamanho de determinadas variáveis morfológicas dos estômatos, como tamanho da célula guarda e do poro estomático (Hetherington \& Woodward 2003). Estômatos menores apresentam poros estomáticos menores, o que propicia respostas mais rápidas a quedas no potencial hídrico foliar. Em espécies de floresta tropical, estômatos maiores são geralmente lentos em suas respostas, o que pode aumentar significativamente a possibilidade de sofrer estresse hídrico em ambientes secos (Aasamaa et al. 2001; Hetherington \& Woodward 2003). 
Neste estudo encontrou-se uma relação significativa entre área do poro estomático e densidade estomática para o conjunto das espécies, desde que a faixa de variação no tamanho do poro esteja entre 0 e $200 \mu \mathrm{m}^{2}$. Nas espécies Guapira noxia e Vochysia thyrsoidea em que o poro estomático cobre uma área acima de $200 \mu \mathrm{m}^{2}$, os valores de condutância estomática foram extremamente baixos. Apesar de o poro estomático ser um fator de fundamental importância no processo de trocas gasosas, outras características estomáticas como a profundidade do poro estomático podem afetar a condutância estomática (Nobel 1999). Estudos mais detalhados são necessários para determinar a influencia das características estomáticas na condutância estomática, especialmente em espécies com estômatos de grandes dimensões.

As relações da densidade de estômatos e condutância estomática e também com a transpiração não foram significativas em nenhum dos casos, demonstrando que para os dois grupos funcionais estudados, a densidade estomática não parece ser fator influente no processo das trocas gasosas. Uma análise da literatura indica que para algumas espécies há uma clara relação positiva entre condutância estomática e densidade estomática (Pearce et al. 2006; Galmes et al. 2007), mas somente na faixa de densidade estomática que vai até 350 estômatos por $\mathrm{mm}^{2}$ (Gratani \& Varone 2004; Galmes et al. 2007). Nem sempre essas relações existem (Abrams 1988), principalmente no cerrado onde o uso de água é limitado por uma combinação de diversos elementos fisiológicos e estruturais das plantas (Goldstein et al. 2008).

O IAE é um índice muito utilizado em estudos de padrões estomáticos, estando muitas vezes correlacionado com a condutância estomática. Este índice é fortemente influenciado pelos valores de densidade estomática, sendo conhecido como bioindicador de mudanças ambientais e na quantidade de chuvas em determinados ambientes (Bongers \& Popma 1990). No entanto, o índice não apresentou diferenças entre cerrado e mata de galeria, sendo um indicativo de semelhanças entre espécies de mata e de cerrado, sugerindo uma possível plasticidade das espécies de mata para adaptação ao ambiente de cerrado. Segundo Cutter (1986) o índice estomático é afetado principalmente pela umidade do ar; assim como as espécies estavam crescendo em condições climáticas muito semelhantes, não houve diferença neste parâmetro.

Os tricomas podem atuar como importantes estruturas de adaptação a ambientes xéricos ou a ambientes sazonais, onde as plantas estão expostas a altos valores de incidência luminosa e baixos valores de umidade relativa, devido a sua capacidade de manter uma atmosfera saturada de vapor de água ao redor da folha pelo aumento da camada de ar limítrofe existente entre a superfície da folha e o ar circundante (Fahn 1986; Fahn \& Cutler 1992; Larcher 2000); além de servir como uma adaptação a altas intensidades luminosas por reduzir a absorção da radiação solar incidente, contribuindo para uma diminuição da temperatura foliar (Ehleringer \& Bjorkman 1978; Klich et al. 1997). Para as espécies aqui estudadas os tricomas parecem não ser elementos adaptativos significativos, pois muitas espécies não o apresentaram, além de não haver uma direção específica neste atributo foliar entre os dois grupos funcionais estudados.

Para a maioria dos parâmetros estomáticos examinados, a maior parte da variação pode ser atribuída a diferenças entre os gêneros. As características estomáticas tendem a ser mais similares entre espécies de uma mesma família do que entre espécies de famílias distintas crescendo num mesmo ambiente (Croxdale 2000). Isto se deve principalmente ao fato de o tamanho do genoma explicar cerca de $60 \%$ das variações no tamanho da célula guarda estomática e da densidade estomática (Beaulieu et al. 2008). Espécies de uma mesma família podem apresentar genomas mais semelhantes do que de outras famílias, o que resultaria em padrões estomáticos bem semelhantes. Desta maneira, a variação entre as espécies dos dois ambientes nos valores de condutância estomática parece ser um produto da historia evolutiva de cada taxa e não o resultado de uma especialização ao ambiente de cerrado ou de mata de galeria.

\section{Agradecimentos}

Os autores agradecem à CAPES, ao CNPq e à NSF (National Sicence Foundation - EUA), pelo apoio financeiro.

\section{Referências bibliográficas}

Aasamaa, K.; Sober, A. \& Rrabi, M. 2001. Leaf anatomical characteristics associated with shoot hydraulic conductance, stomatal conductance and stomatal sensitivity to changes of leaf water status in temperate deciduous trees. Australian Journal of Plant Physiology 28: 765-774.

Abrams, M.D. 1988. Genetic variation in leaf morphology and plant and tissue water relations during drought in Cercis Canadensis L. Forest Science 34: 200-207.

Abrams, M.D.; Kubiske, M.E. \& Mostoller, S.A.1994 Relating wet and dry year ecophysiology to leaf structure in contrasting temperate tree species. Ecology 75: 123-133

Ashton, P.M.S. \& Berlyn, G.P. 1994. A comparison of leaf physiology and anatomy of Quercus (section Erythrobalanus - Fagaceae) species in different light environments. American Journal of Botany 81: 589-587.

Beaulieu, J.M.; Leitch, I.J.; Patel, S.; Pendharkar, A. \& Knight, C.A. 2008. Genome size is a strong predictor of cell size and stomatal density in Angiosperms. New Phytologist 179: 975-986. 
Boeger, M.R.T. \& Wisniewski, C. 2003. Comparação da morfologia foliar de espécies arbóreas de três estádios sucessionais distintos de floresta ombrófila densa (Floresta Atlântica) no Sul do Brasil. Revista Brasileira de Botânica 26: 61-72.

Bongers, F. \& Popma, J. 1990. Leaf characteristics of the tropical rainforest flora of Los Tuxtlas, Mexico. Botanical Gazette 151: 354-365.

Carswell, F.E.; Meier, P.; Wandelli, E.V.; Bonates, L.C.M.; Kruijt, B.; Barbosa, E.M.; Nobre, A.D.; Grace, J. \& Jarvis, P.G. 2000. Photosynthetic capacity in central Amazonian rain forest. Tree Physiology 20: 179-186.

Cole, M.M. 1992. Influence of physical factor on the nature and dynamics of forest-savanna boundaries. Pp. 63-75. In: P.A. Furley.; J. Proctor \& J. Ratter (eds.). Nature and Dynamics of ForestSavanna Boundaries. London, Chapman and Hall.

Coutinho, L.M. 1978. O conceito de cerrado. Revista Brasileira de Botânica 1: 17-23.

Croxdale, J.L. 2000. Stomatal patterning in Angiosperms. American Journal of Botany 87: 1069-1080.

Cutter, E.G. 1986. Anatomia vegetal. Parte I - Células e tecidos. 2 ed. São Paulo, Roca.

Dunlapp, J.M. \& Stettler, R.F. 2001. Variation in leaf epidermal and stomatal traits of Populus trichocarpa from two transects across the Washigton Cascades. Canadian Journal of Botany 79: 528-536.

Ehleringer, J.R. \& Bjorkamann, O. 1978. Pubescence and leaf spectral characteristics in a desert shrub, Encelia farinosa. Oecologia 37: $183-200$

Fahn, A.1986 Structural and functional properties of trichomes of xeromorphic leaves. Annals of Botany 57: 631-637

Fahn, A. \& Cutler, D. 1992. Xerophytes. Berlin, Gebruder Borntraeger.

Felfili, J.M. \& Silva Junior, M.C.D. 1992. Floristic composition, phytosociology and comparison of cerrado and gallery forests at Fazenda Agua Limpa, Federal District, Brazil. Pp. 393-416. In: P.A. Furley; J. Procter \& J.A. Ratter (eds.). Nature and Dynamics of Forest-Savanna Boundaries. London, Chapman \& Hall.

Franco, A.C. 2002. Ecophysiology of woody plants. Pp. 178-197. In: P.S. Oliveira \& R.J. Marquis (eds.). The cerrados of Brazil. New York, Columbia University Press.

Franklin, G.L. 1945. Preparation of thin sections of synthetic resins and wood-resin composites, and a new macerating method for wood. Nature 155: 51.

Galmes, J.; Flexas, J.; Savé, R. \& Medrano, H. 2007. Water relations and stomatal characteristics of Mediterranean plants with different growth forms and leaf habits: responses to water stress and recovery. Plant Soil 290: 139-155.

Givnish, T.J. 1984 Leaf and canopy adaptations in tropical forests. Pp. 51-84. In: E. Medina; H.A. Mooney \& C. Vasques-Yanes (eds.) .Physiological ecology of plants in the wet tropics. Dr. W.Junk Publishers, The Hague.

Goodland, R. \& Pollard, R. 1973. The Brazilian cerrado vegetation: a fertility gradient. Journal of Ecology 61: 219-224.

Golstein, G.; Meinzer, F.C.; Bucci, S.J.; Scholz, F.G.; Franco, A.C. \& Hoffmann, W.A. 2008. Water economy of Neotropical savanna trees: six paradigms revisited. Tree Physiology 28: 395-404.

Gratani, L. \& Varone, L. 2004. Adaptative photosynthetic strategies of Mediterranean maquis species according to their origin. Photosynthetica 42: 551-558.

Hetherington, A.M. \& Woodward, F.I. 2003. The role of stomata in sensing and driving environmental change. Nature 424: 901-908.

Hoffmann, W.A. 2000. Post-establishment seedling success in the Brazilian cerrado: A comparison of savanna and forest species. Biotropica 32: 62-69.

Hoffmann, W.A. \& Franco, A.C. 2003. Comparative growth analysis of tropical Forest and savanna woody plants using phylogeneticallyindependent contrasts. Journal of Ecology 91: 475-484.

Hoffmann, W.A.; Orthen, B. \& Franco, A.C. 2004. Constraints to seedling success of savanna and forest trees across the savannaforest boundary. Oecologia 140: 252-260.
Hoffmann, W.A.; Franco, A.C.; Moreira, M.Z. \& Haridasan, M. 2005. Specific leaf area explains differences in leaf traits between congeneric savanna and forest trees. Functional Ecology 19: 932-940.

Jackson, P.C.; Meinzer, F.C.; Bustamante, M.; Goldstein, G.; Franco, A.C.; Rundel, P.W.; Caldas, L.; Igler, E. \& Causin, F. 1999. Partitioning of soil water among tree species in Brazilian cerrado ecosystem. Tree Physiology 19: 717-724.

Justo, C.F.; Soares, A.M.; Gavilanes, M.L. \& Castro, E.M. 2005. Leaf anatomical plasticity of Xylopia brasiliensis Sprengle (Annonaceae). Acta Botanica Brasilica 19: 111-123.

Klich, M.G.; Brevedan, R.E. \& Villamil, S.C. 1997, Leaf anatomy and ultrastructure of Poa ligularis after defoliation and water strees. Proceedings of the $18^{\text {th }}$ International Grassland Congress 1: $37-38$

Larcher, W. 2000. Ecofisiologia vegetal. São Carlos, Ed. Rima.

Ledru, M.P. 2002. Late quaternary history and evolution of the cerrados revealed by palynological records. Pp. 33-50. In: P.S. Oliveira \& R.J. Marquis (eds.). The cerrados of Brazil. New York, Columbia University Press.

Lleras, E. 1974. Differences in stomatal number per unit are within the same species under different micro-environmental conditions: A working hypothesis. Acta Amazonica 7: 473-476.

Marques, A.R.; Garcia, Q.S.; Passos Rezende, J.L. \& Fernandes, G.W. 2000. Variations in leaf characteristics of two species of Miconia in the Brazilian cerrado under different light intensities. Tropical Ecology 41: 47-56.

Mishra, M.K. 1997. Stomatal characteristics at different ploidy levels in Coffea L. Annals of Botany 80: 689-692.

Nobel, P.S. 1999. Physicochemical \& environmental plant physiology. $2^{\text {nd }}$ ed. San Diego, Academic Press.

Oliveira Filho, A.T. \& Ratter, J.A. 2002. Pp. 121-140. Vegetation physiognomies and woody flora of the Cerrado Biome In: P.S. Oliveira \& R.J. Marquis (eds.). The cerrados of Brazil. New York, Columbia University Press.

Pandey, S. \& Nagar, P.K. 2003. Patterns of leaf surface wetness in some important medicinal and aromatic plants of Western Himalaya. Flora 198: 349-357.

Pearce, D.W.; Millard, S.; Bray, D.F. \& Rood, S.B. 2006. Stomatal characteristics of riparian poplar species in a semi-arid environment. Tree Physiology 26: 211-218.

Pillar, V.D. \& Sosinski Jr., E. 2003. An improved method for searching plant functional types by numerical analysis. Journal of Vegetation Science 14: 323-332.

Prado, C.H.B.A.; Wenhui, Z.; Rojas, M.H.C. \& Souza, G.M. 2004. Seasonal leaf gás exchange and water potential in woody cerrado species community. Brazilian Journal of Plant Physiology 16: $7-16$

Rawitscher, F. 1948. The water economy of the vegetation of the campos cerrados in southern Brazil. Journal of Ecology 36: 237-267.

Scholz, F.G.; Bucci, S.J.; Goldstein, G.; Meinzer, F.C. \& Franco, A.C. 2002. Hydraulic redistribution of soil water by neotropical savanna trees. Tree Physiology 22: 603-612.

Shields, L.M. 1950. Leaf xeromorphy as related to physiological and structural influences. Botanical Review 19: 399-447.

Silva, L.C.R.; Sternberg, L.; Haridasan, M.; Hoffmann, W.A.; MirallesWilhelm, F. \& Franco, A.C. 2008. Expansion of gallery forests into central Brazilian savannas. Global Change Biology 14: 2108-2118.

Smith, W.K.; Bell, D.T. \& Sheperd, K.A. 1998. Associations between leaf structure, orientation and sunlight exposure in five Western Australian communities. American Journal of Botany 85: 56-63.

Sparks, J.P. \& Black, A. 1999. Regulation of water loss in populations of Populus trichocarpa: the role of stomatal control in preventing xylem cavitation. Tree Physiology 19: 453-459.

Zar, J.H. 1999. Biostatistical Analysis. $4^{\text {th }}$ ed. New Jersey, Prentice Hall, Englewood Cliffs. 